International Journal on Integrating Technology in Education (IJITE) Vol.6, No.4, December 2017

\title{
AN INTERACTIVE ONLINE TRAINING COURSE FOR SQL BEGINNERS
}

\author{
Carlos R. Jaimez-González, Arturo Palma-Hernández \\ Departamento de Tecnologías de la Información \\ Universidad Autónoma Metropolitana, Unidad Cuajimalpa, México
}

\begin{abstract}
Information and Communication Technologies have been used to support education in several levels, due to the need of new ways to access learning resources. Some representative examples of these new learning resources are online training courses, which guide the learning process of students through a series of activities to be carried out by themselves. This paper presents an interactive online training course for learning SQL, which is a widely used language for relational database management. The online course allows students to learn SQL through explanations and interactive examples, which are executed by an interpreter, and the results of the executions are visualized directly on the same online course. All registered students have their own database created dynamically, which gives them freedom of modifying the examples of the training course to experiment with different SQL sentences, without affecting other students.
\end{abstract}

\section{KEYWORDS}

ICT for Education, Online Training Course, Relational Databases, SQL, Interactive Course.

\section{INTRODUCTION}

Information and Communication Technologies (ICT) have been used by educational institutions and organizations for offering online training courses [1], as a response for new ways of learning. Online training courses guide the learning process of students through a series of activities to be carried out by themselves $[2,3]$.

This paper presents an interactive online training course for learning the Structured Query Language (SQL), which is part of the syllabus of an introductory databases course that is taught at the Information Technologies and Systems Undergraduate program at our faculty. The online training course guides students through a series of topics and examples that cover SQL basics.

The rest of the paper is organized as follows. Section 2 describes the motivation to develop this project. Section 3 presents a review of existing web sites that offer online SQL training courses or tutorials, with a description of their main features, and a comparison of them. The design and implementation of the interactive online training course is presented in section 4 , along with the technologies, tools and languages employed, the topics covered and the sample database used by the training course. Section 5 shows the web interface of the training course, with a description of its organization. Finally, section 6 presents conclusions and future work.

\section{Motivation}

Information systems require programming languages to be implemented, such as Java, C\#, Python, among others. On the other hand, the databases that support these information systems require query languages to access and modify data, such as SQL, which is a declarative programming language to access relational databases; it allows to invoke different types of operations on the data stored. 
International Journal on Integrating Technology in Education (IJITE) Vol.6, No.4, December 2017

SQL provides two languages: the Data Definition Language (DDL), and the Data Manipulation Language (DML). The DDL is used to specify a database scheme using its three basic operations on database objects: the CREATE sentence allows to generate new objects in the database, such as tables, views, stored procedures, triggers, etc.; the ALTER sentence modifies the objects that are already in the database; and the DROP sentence deletes existing objects from the database. The DML is used to execute operations on the database, which affect directly the data on tables: the SELECT sentence allows to query one or several tables with some conditions; the INSERT sentence is used to add a record to a specific table; the UPDATE sentence allows to modify one or more records of a specific table; and the DELETE sentence deletes one or more records from a specific table.

Currently, several products for development of information systems are compatible with SQL, which has been established as an standard language for accessing and modifying data in relational databases [4, 5]. SQL is positioned in the top places as a language for implementation and administration of relational databases [6].

In order to master a programming language, it is needed to carry out a lot of practice with several examples, which have to be done normally outside the classroom as an additional activity. There are training courses and tutorials on the web for practicing different technologies and programming languages; most of them come with explanations, but a limited set of examples are presented [3]. There are also some other courses that do not have enough content for the comprehension of specific topics; and also they do not provide any interactive way of studying their contents.

The constant practice of a programming language allows students to master it; for this reason in this paper it is presented an interactive online training course, which has been developed to support the learning of SQL for relational databases courses. One of the key features of this training course is that it allows the teacher to create its own SQL examples and exercises, which can be executed by students through an SQL interpreter directly when they study the course, without needing to install any database management system on the computer where they visualize the online training course. Additionally, students can freely modify the examples of the training course to experiment with different SQL sentences on a database created dynamically for each student.

It should be noted that the first author has been involved in the design and implementation of some web tools to support teaching and learning of different subjects, such as object oriented programming [7], structured programming [8, 9], databases [10], web programming [11], data structures [12], among others.

\section{RELATED WORK}

This section describes the functionality and the main features of different existing web sites that contain training courses or tutorials for SQL, which were analysed in this paper: W3Schools [13], SQL Course [14], 1Keydata [15], WebCheatSheet [16], Tutorialspoint [17].

\subsection{W3SCHOOLS}

W3Schools [13] is a web site that offers tutorials for different languages for web development, such as HTML, CSS, JavaScript, PHP, JQuery, SQL, among others. In the case of the SQL tutorial, students can study topics of DDL and DML through some examples, which can be executed with an interpreter included in the web site. Additionally, there are examples of SQL functions, a language reference section, and an evaluation with 20 questions. W3Schools does not require registration, it is a free web site, and all tutorials are in English. 
International Journal on Integrating Technology in Education (IJITE) Vol.6, No.4, December 2017

\subsection{SQL COURSE}

SQL Course [14] is a web site that provides an introductory SQL training course, which allows to practice with examples that can be executed through an SQL interpreter. Some of the topics that SQL Course offers are the following: basic tables, creating tables, inserting into a table, updating records, deleting records, drop a table, aggregate functions, order by clause, group by clause, having clause, among others. SQL Course can be accessed freely, it does not require to create any user account for solving the exercises, and all its contents are in English.

\subsection{KEYDATA}

1KeyData [15] is a web site with its contents in English, free access, that offers information and tutorials for programming and data storage. Some of the programming languages and web technologies that can be found on this web site are the following: SQL, HTML, CSS, PHP, etc. The SQL tutorial has a list of the most used sentences, and they are divided in the following lessons to be studied: SQL Commands; SQL Functions; SQL Join; SQL String Functions; SQL Date Functions; Data Definition Language (DDL); SQL Constraints; SQL Alter Table; SQL Null; Advanced SQL; SQL Syntax; SQL Quiz.

A lesson in 1KeyData contains an explanation of the SQL sentence, then the syntax of the sentence is shown, next an example is executed with the SQL interpreter included. The web site has a few examples for every sentence and it shows the result obtained on a database given.

\subsection{WEBCHEATSHEET}

WebCheatSheet [16] is a web site written in English, with free registration, focused on tools for databases, tutorials and technical papers. It offers information on web technologies, such as ASP, PHP, JavaScript, HTML, SQL, among others. The SQL tutorial presents a menu with the basic sentences, which cover SQL data types, SELECT statement, UPDATE statement, DELETE statement, INSERT statement, DISTINCT, IN, LIKE, ORDER BY, GROUP BY, HAVING, JOIN, BETWEEN, AND, OR, ALTER TABLE, etc. The web site has a SQL interpreter that allows to practice the SQL sentences, and the results are shown after execution.

\subsection{TUTORIALSPOINT}

Tutorialspoint [17] is a web site of free access, in English, which provides an introduction to the SQL basic sentences. In the index page of Tutorialspoint, there is menu that displays the topics offered: SQL Overview; Relational Database Management Concepts; Databases; Syntax; Data Types; Operators; Expressions; Create Database; Drop Database; Select Database; Create Table; Drop Table; Insert Query; Select Query; among others.

This web site provides examples for each topic, where the correct syntax is first shown, then an example with the SQL code is presented. It should be noted that this web site is static, which means that it does not have any interpreter for executing SQL sentences; it does not have user registration; there is no evaluation for students.

\subsection{COMPARISON}

Table 1 shows a comparison of features among the five web sites mentioned in the previous sections: T1) W3Schools, T2) SQL Course, T3) 1Keydata, T4) WebCheatSheet, and T5) Tutorialspoint; the tick indicates that the web site has the feature, and the cross indicates that the web site does not have it. After presenting Table 1, it is also provided a brief description of the features considered, which were used to evaluate the web sites analysed. 
International Journal on Integrating Technology in Education (IJITE) Vol.6, No.4, December 2017

Table 1. Features to evaluate the web sites analysed.

\begin{tabular}{|l|c|c|c|c|c|}
\hline \multicolumn{1}{|c|}{ Features } & T1 & T2 & T3 & T4 & T5 \\
\hline SQL code fragments & $\checkmark$ & $\checkmark$ & $\mathbf{x}$ & $\checkmark$ & $\checkmark$ \\
\hline SQL interpreter & $\checkmark$ & $\checkmark$ & $\mathbf{x}$ & $\checkmark$ & $\mathbf{x}$ \\
\hline Evaluation & $\checkmark$ & $\mathbf{x}$ & $\checkmark$ & $\mathbf{x}$ & $\mathbf{x}$ \\
\hline Database change & $\mathbf{x}$ & $\mathbf{x}$ & $\mathbf{x}$ & $\mathbf{x}$ & $\mathbf{x}$ \\
\hline User accounts & $\mathbf{x}$ & $\mathbf{x}$ & $\mathbf{x}$ & $\mathbf{x}$ & $\mathbf{x}$ \\
\hline DML execution & $\checkmark$ & $\checkmark$ & $\mathbf{x}$ & $\checkmark$ & $\mathbf{x}$ \\
\hline DDL execution & $\mathbf{x}$ & $\mathbf{x}$ & $\mathbf{x}$ & $\mathbf{x}$ & $\mathbf{x}$ \\
\hline
\end{tabular}

SQL code fragments. This feature means that the web site or tutorial includes static examples (only text) of the code syntax for the SQL sentences presented as part of the explanations.

SQL interpreter. This feature refers to having an area where the student can write SQL sentences, which can be executed, and the result of the execution can be visualized immediately.

Evaluation. This feature indicates that the tutorial or web site includes a way of feedback for the student, through a multiple choice questionnaire.

Database change. This feature allows the tutorial or web site to change the working database, on which the student executes SQL sentences.

User accounts. This feature allows students to have their own user account, which can be used to review the topics studied and the evaluations taken.

DML execution. This feature means that the SQL interpreter supports the execution of the data manipulation language sentences, such as INSERT, UPDATE, DELETE, and SELECT.

DDL execution. This feature means that the SQL interpreter supports the execution of the data definition language sentences, such as CREATE, ALTER, and DROP.

\section{ImPlementation Of The Online Training Course}

After analysing the different web sites and tutorials that were described in the previous section, the implementation of the online training course presented in this paper has all the features listed in Table 1.

In this online training course it is possible to study the SQL basic topics; it is a web site that integrates important features, such as the module for execution of SQL sentences, where students can interact with the course through an SQL interpreter, which executes SQL sentences on a database and visualizes the result of the execution immediately on the online course.

The training course requires the student to register and create its own user account, which allows to study the SQL topics included, such as the DDL and DML. Each student will have its own database on which it is possible to execute queries and other SQL sentences. Additionally, students can take an examination with multiple choice questions, which is an informative evaluation with the purpose of giving feedback to them in their process of learning.

\subsection{TeChnologies, ToOls And LANgUages USED}

The implementation of the interactive online training course involves the following technologies, tools and languages: the Hypertext Markup Language (HTML) for the interface of the web site and its contents; the Cascading Style Sheets (CSS) for the presentation of the web site; JavaScript is used for validations of HTML controls, and for incorporating interactivity in the web site; the 
International Journal on Integrating Technology in Education (IJITE) Vol.6, No.4, December 2017

database management system MySQL for storing and accessing data; Java for creating business classes for processing and accessing data; Java Server Pages (JSP) for the dynamic generation of interfaces and access to Java classes.

\subsection{Topics Covered In The Training Course}

The DML topics covered by the online training course are the following: SELECT for demonstrating the use of queries; INSERT for inserting records into a table; UPDATE for showing how to update records of a table; DELETE for demonstrating how to delete records from a table; AGGREGATION for the aggregation operations; SETS for operations on sets; ORDERING for demonstrating the use of the ORDER BY clause; STRINGS for showing the operations available on strings.

The DDL topics included in the training course are the following: CREATE for creating objects on the database; ALTER for modifying objects on the database; DROP for eliminating objects from the database. Additionally, the online training course provides a topic that covers the creation of VIEWS, and a section for an EVALUATION of the student.

\subsection{SAMPLE DATABASE}

The topics covered by the training course use a sample database, which is created dynamically for each student that takes the training course. All the SQL sentences written in the SQL interpreter are executed on an instance of the database shown in Figure 1, which represents a database for a university enrollment system, composed of seven tables: alumno, grupoalumno, иea, grupo, profesor, departamento, and trimestre. The explanations and examples included in the training course as part of the topics, use this database to execute SQL sentences.

\section{Interface Of The Online Training Course}

This section shows some screenshots of the interactive online training course for SQL. It should be noted that the web interface presented is a preliminary version, and there is still work to be done to modify its design and appearance.

Figure 2 shows a screenshot of the online training course after having selected the SELECT topic on the left menu. The training course is divided in different SQL topics, which are listed on the left panel, such as SELECT, INSERT, UPDATE, DELETE, AGGREGATION, CREATE, ALTER, DROP, among others. The name of the student logged into the training course is shown in the right corner of the top panel. The right panel shows the sample database used in the training course, which has the seven tables shown in Figure 1: alumno, grupoalumno, uea, grupo, profesor, departamento, and trimestre; each table has a number of sample records to be used in the explanations and examples throughout the training course, so the student can modify its own database by executing DDL and DML sentences. Finally, the centre panel shows an example of the SELECT sentence that can be executed by clicking the Ejecutar (Execute) button, which triggers the SQL interpreter. 
International Journal on Integrating Technology in Education (IJITE) Vol.6, No.4, December 2017

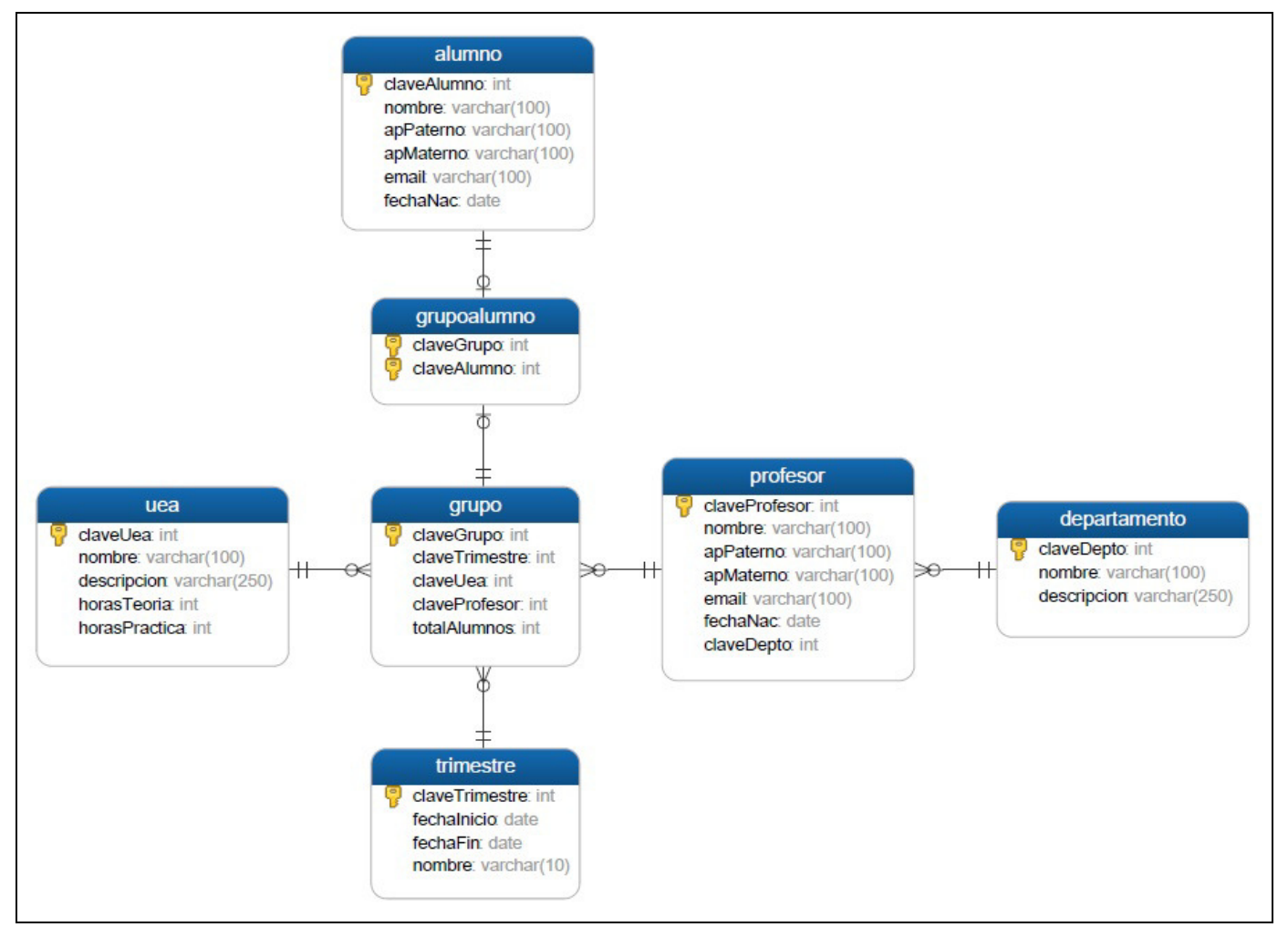

Figure 1. Database diagram for the sample database used in the training course

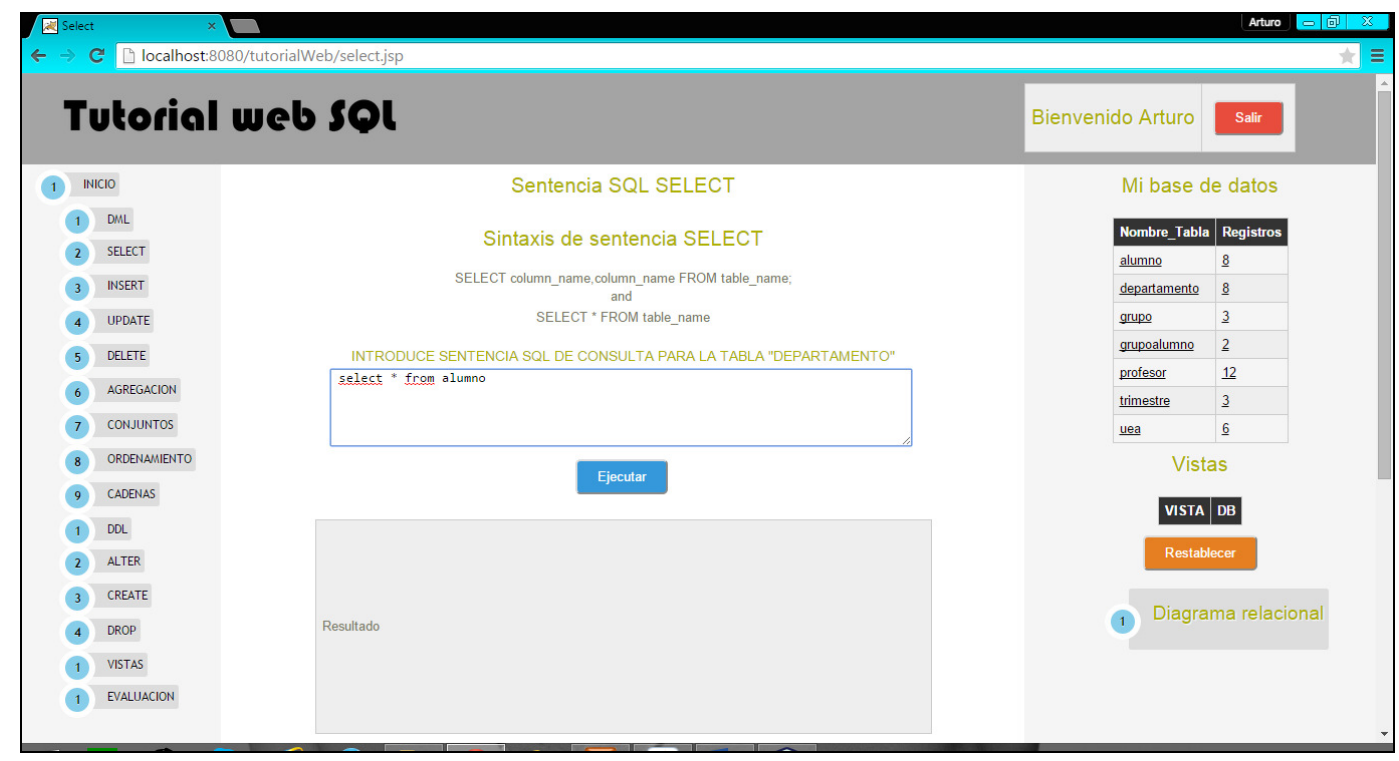

Figure 2. The interactive online training course for SQL

Figure 3 shows a screenshot of the online training course after having executed the SQL sentence SELECT * FROM alumno on the sample database; it shows the results in the grey panel below the text area, where it can be observed that it retrieves all the fields and records from the alumno table, which has 6 fields, and 7 records. The execution of these sentences is asynchronous, using AJAX, which avoids refreshing the complete web page. 
International Journal on Integrating Technology in Education (IJITE) Vol.6, No.4, December 2017

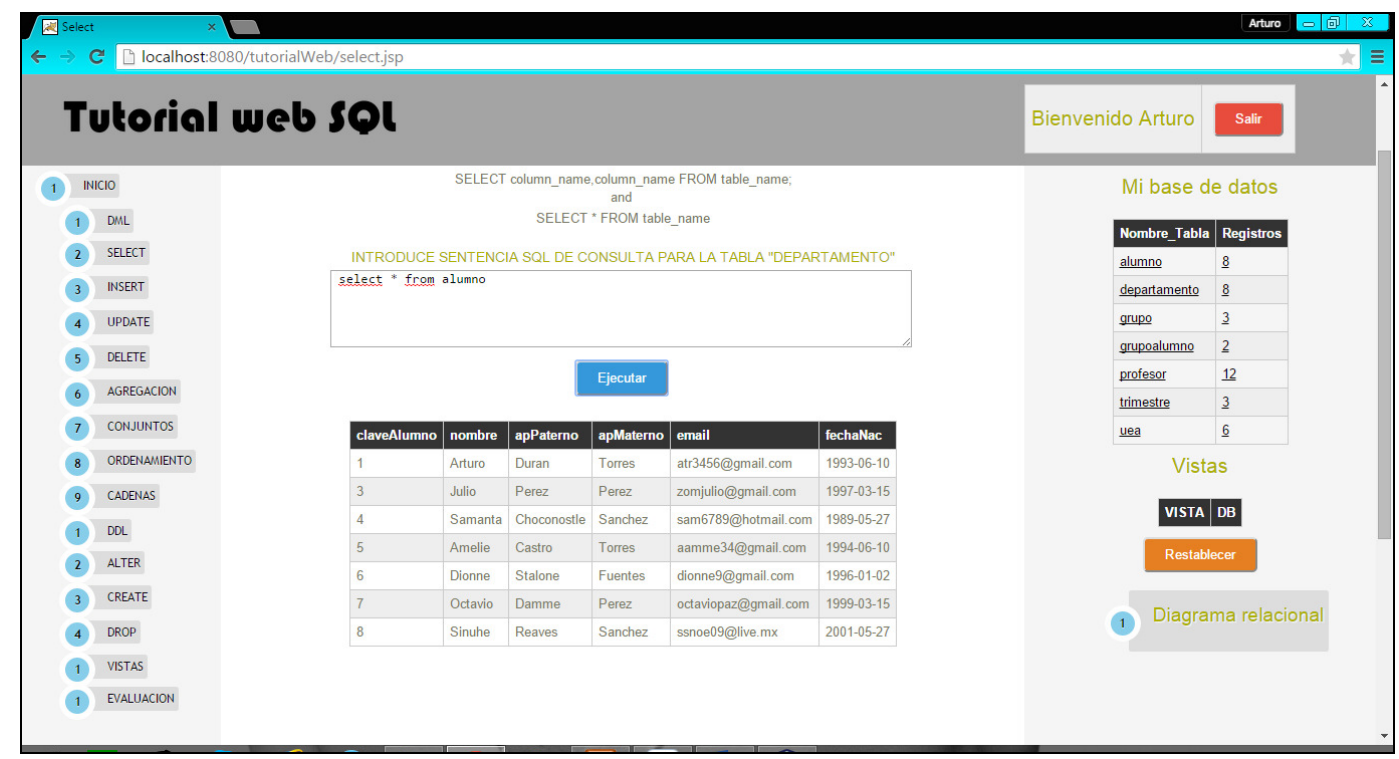

Figure 3. The SQL interpreter executing a SELECT sentence and showing results

The execution of DDL sentences is similar to the execution to DML sentences. For example, Figure 4 shows the web page with the CREATE topic, where there is also a text area for writing SQL sentences in the interpreter; in this case the SQL code written in the interpreter represents the creation of the Prueba table, with five fields, as follows: CREATE TABLE Prueba( PruebaID int, nombre varchar(255), apellido varchar (255), direccion varchar (255), Ciudad varchar (255)); .

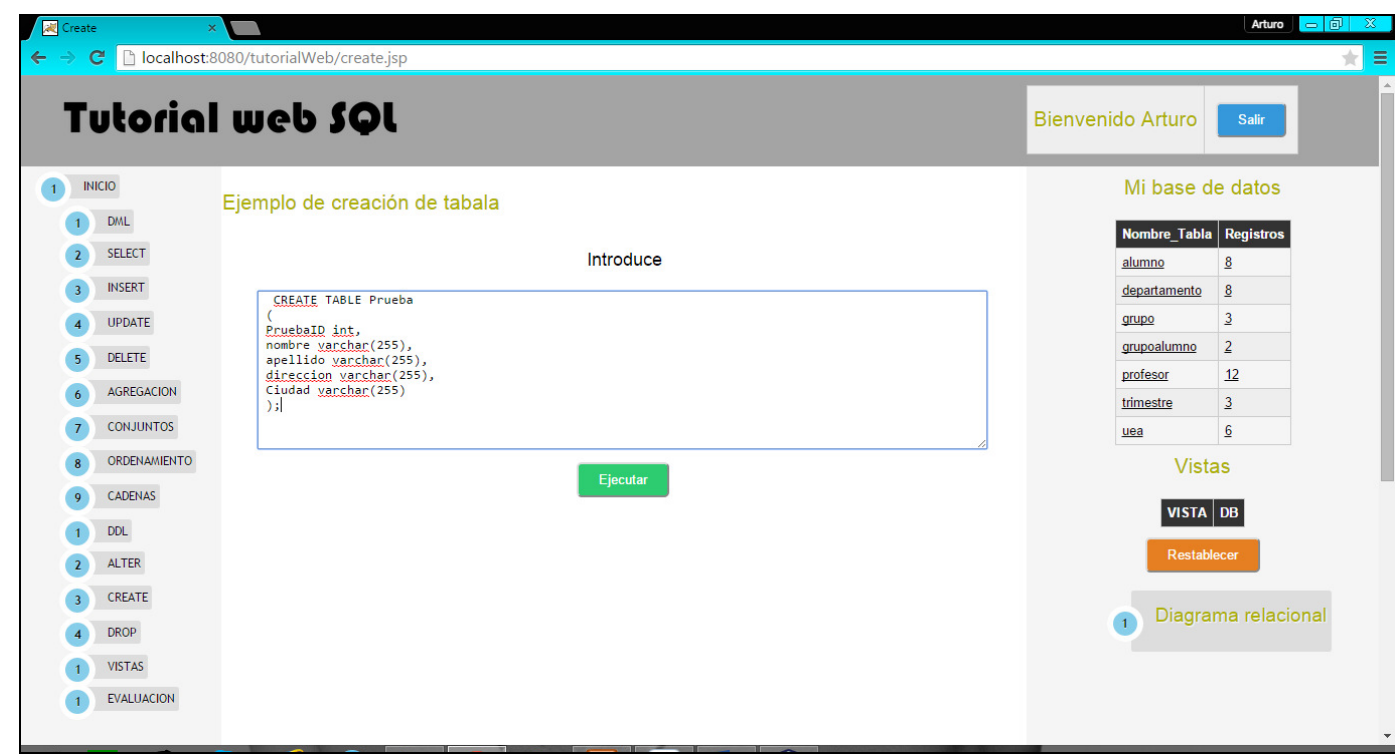

Figure 4. The CREATE sentence in the SQL interpreter

Figure 5 shows the execution of the CREATE sentence, which creates the Prueba table on the student database; this can be verified on the database visualized on the right panel, where the Prueba table is shown in the sixth place, just below the Profesor table. It can be noticed that the table is recently created, because it has 0 registries. The execution of these sentences is also through asynchronous communication with AJAX, which avoids refreshing the web page. 


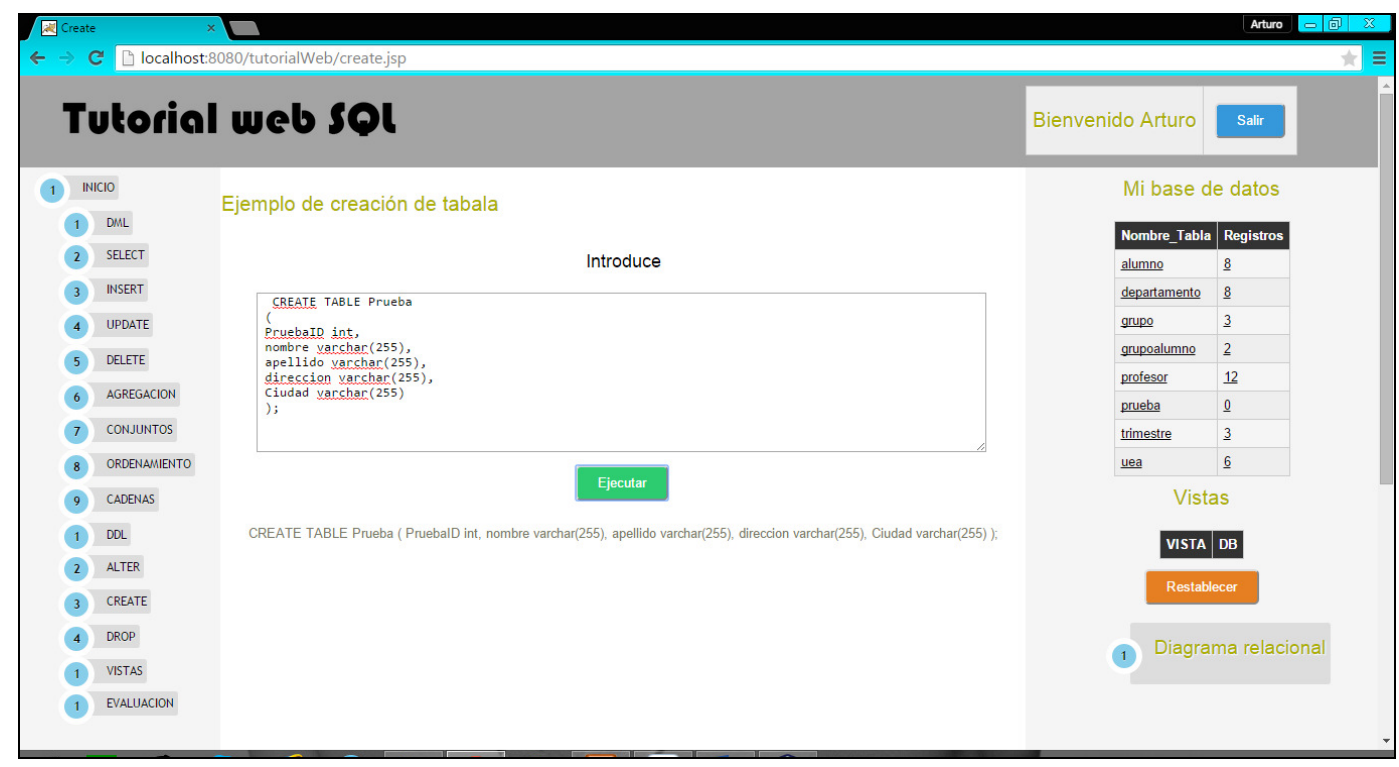

Figure 5. Creation of a new table and visualization of the table on the right panel

\section{CONCLUSiOnS AND Future Work}

This paper presented an interactive online training course for SQL, which guides students through the set of topics and activities that cover SQL. The training course allows students to register in order to study the DDL and DML topics through explanations and examples, which can be modified and executed through an SQL interpreter, and visualize the results of the execution immediately, without needing to install any database management system on the computer where they visualize the online training course. Other key feature of this training course is that it allows the teacher to create its own SQL examples and exercises. Additionally, the training course has an evaluation with multiple choice questions for measuring the performance of students.

It was also presented a comparison of different existing web sites that contain training courses or tutorials for SQL, where some important features were analysed. None of the existing training courses examined allow the execution of DDL sentences, which is a disadvantage because students are not able to practice these types of sentences. The interactive online training course presented in this paper supports the execution of both types of sentences: DML for manipulating data and DDL for defining data objects.

Further work is needed to complete the contents of the training course, which will include other DDL and DML sentences, such as those for creating and manipulating views, triggers and stored procedures. It is also needed to improve its user interface design, and carry out some usability tests with teachers and students of database undergraduate courses, as well to develop an evaluation instrument to be applied to them.

\section{REFERENCES}

[1] Area, M. (2001). La oferta de educación superior a través de Internet. Análisis de los campus virtuales de las universidades españolas. Informe final. Dirección General de Universidades. Ministerio de Educación, Cultura y Deporte. http://www.edulab.ull.es/campusvirtuales.

[2] Jaimez-González, C. R., Sánchez-Sánchez, C., Zepeda-Hernández, S. (2011). Creating and Administering Interactive Online Tutorials and Performance Evaluation Tests Through a Novel Web Platform. International Journal for Cross-Disciplinary Subjects in Education (IJCDSE), 2(3), 447-455. 
International Journal on Integrating Technology in Education (IJITE) Vol.6, No.4, December 2017

[3] García, L. (2001). La Educación a distancia. De la teoría a la práctica. Barcelona: Ariel, http://www.edulab.ull.es/campusvirtuales.

[4] Silberschatz, A. (2002). Fundamentos de bases de datos. Madrid, Spain: McGraw-Hill.

[5] Ozsu, L., Tamer, M. (2009). Structured Query Language. Encyclopedia of Database Systems. USA: Springer.

[6] Microsoft SQL Server. (2010). http://elpaladintecnologico.blogspot.mx/2010/07/microsoft-sql-servery-el-mundo-laboral.html.

[7] Jaimez-González, C. R., Luna-Ramírez, W. (2013). Towards a Web Learning Environment for Supporting Object Oriented Programming Courses. Journal of Research in Computing Science, Advances in Computing Science, 67, 33-40.

[8] Jaimez-González, C. R., Luna-Ramírez, W. (2014). Supporting Structured Programming Courses Through a Set of Learning Objects. Proceedings of the IEEE International Conference on Information Society, 124-128, London, UK.

[9] Castillo-Cortes, M., Jaimez-González, C. R. (2016). Hacia una Herramienta Web para Visualizar la Ejecución de Programas Escritos en el Lenguaje Java. Revista Research in Computing Science, 125, 109-120.

[10] Martínez-Samora, J., Jaimez-González, C. R. (2016). Propuesta de aplicación educativa en línea para crear diagramas E-R. Revista Iberoamericana de Producción Académica y Gestión Educativa, (JulioDiciembre).

[11] Jaimez-González, C. R., Vargas-Rodríguez R. (2017). Editor web visual para HTML, CSS y JavaScript de apoyo a la docencia. Virtualidad, Educación y Ciencia, 8 (14), 136-152.

[12] García-Mendoza, B., Ruíz-Mendoza, P., Real-Flores, G., Jaimez-González, C. R., Villatoro-Tello, E. (2015). Diseño e Implementación de Applets como Material Didáctico de Apoyo para Cursos de Estructuras de Datos. Revista Pistas Educativas, 112, 530-551.

[13] Refsnes Data. W3Schools SQL Tutorial, http://www.w3schools.com/sq1/default.asp.

[14] QuinStreet Inc. (2015). Interactive Online SQL Training. http://www.sqlcourse.com/.

[15] 1keydata.com. (2015) SQL Tutorial. http://www.1keydata.com/sql/sql.html.

[16] WebCheatSheet. (2014). SQL Tutorial. http://webcheatsheet.com/sql/interactive_sql_tutorial/.

[17] Tutorialspoint. SQL Tutorial Databases. http://www.tutorialspoint.com/sql/.

\section{AUTHORS}

Carlos R. Jaimez-González is an Associate Professor at the Information Technologies Department at the Universidad Autónoma Metropolitana Campus Cuajimalpa, in Mexico City. He received his $\mathrm{PhD}$ degree in Computer Science at the University of Essex, United Kingdom in 2011. His research interests include Technologies for Supporting Education, Interoperability in Distributed Systems, XML and Related Technologies, and the Development of Web and E-Commerce Applications. He has a distinction as a National Researcher from the Mexican Government.

Arturo Palma-Hernández is studying the final year of the Information Technologies and Systems undergraduate program at the Universidad Autónoma Metropolitana Campus Cuajimalpa, in Mexico City. His research interests include Technologies for Supporting Education, and the Development of Web and E-Commerce Applications.
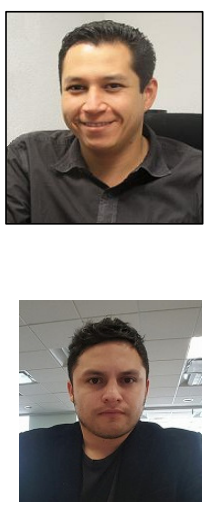